\title{
The 99th Fibonacci Identity
}

\author{
Arthur T. Benjamin, Alex K. Eustis, and Sean S. Plott \\ Department of Mathematics \\ Harvey Mudd College, Claremont, CA, USA \\ benjamin@hmc.edu
}

Submitted: Feb 7, 2007; Accepted: Jan 30, 2008; Published: Feb 25, 2008

Mathematics Subject Classification: 05A19, 11B39

\begin{abstract}
We provide elementary combinatorial proofs of several Fibonacci and Lucas number identities left open in the book Proofs That Really Count [1], and generalize these to Gibonacci sequences $G_{n}$ that satisfy the Fibonacci recurrence, but with arbitrary real initial conditions. We offer several new identities as well. Among these, we prove $\sum_{k \geq 0}\left(\begin{array}{l}n \\ k\end{array}\right) G_{2 k}=5^{n} G_{2 n}$ and $\sum_{k \geq 0}\left(\begin{array}{l}n \\ k\end{array}\right) G_{q k}\left(F_{q-2}\right)^{n-k}=\left(F_{q}\right)^{n} G_{2 n}$.
\end{abstract}

In the book Proofs that Really Count [1], the authors use combinatorial arguments to prove many identities involving Fibonacci numbers, Lucas numbers, and their generalizations. Among these, they derive 91 of the 118 identities mentioned in Vajda's book [2], leaving 27 identities unaccounted. Eight of these identities, presented later in this paper, have such a similar appearance, the authors remark (on page 144) that "one good idea might solve them all." In this paper, we provide elegant combinatorial proofs of these Fibonacci and Lucas identities along with generalizations to arbitrary initial conditions.

Before examining these new identities, we warm up with the following well known identity, which will allow us to define terminology and illustrate our approach.

Identity 1. For $n \geq 0$,

$$
\sum_{k \geq 0}\left(\begin{array}{l}
n \\
k
\end{array}\right) F_{k}=F_{2 n}
$$

Here, the Fibonacci numbers $F_{n}$ have initial conditions $F_{0}=0, F_{1}=1$, and this identity has an elementary algebraic proof (using Binet's formula and the binomial theorem), but it also has a completely transparent proof using the combinatorially defined Fibonacci number $f_{n}=F_{n+1}$.

It is easy to show (as in [1]) that $f_{n}$ counts the ways to tile a one-dimensional board of length $n$ using squares of length one and dominoes of length two. We refer to such tilings 
as $n$-tilings and express these tilings using the notation $d$ for domino and $s$ for square. For example, the 9-tiling in Figure 1 can be represented as $d s d d s s$ or $d s d^{2} s^{2}$.

\begin{tabular}{|c|c|c|c|c|c|}
\hline 12 & 3 & 5 & 6 & 8 & 9 \\
\hline d & $s$ & d & d & $s$ & $s$ \\
\hline
\end{tabular}

Figure 1: The 9-tiling $d s d^{2} s^{2}$

With this in mind, we can now prove Identity 1 rewritten as

Identity 1. For $n \geq 0$,

$$
\sum_{k \geq 0}\left(\begin{array}{l}
n \\
k
\end{array}\right) f_{k-1}=f_{2 n-1} .
$$

Proof: Let $S$ denote the set of $(2 n-1)$-tilings. The size of $S$ is $f_{2 n-1}$, which is counted by the right side of Identity 1 . The left side of the identity counts the same set $S$ by considering the number of square tiles among the first $n$ tiles. (Note that any tiling of length $2 n-1$ must have at least $n$ tiles.) To create a $(2 n-1)$-tiling with $k$ squares among the first $n$ tiles, we first choose which of the first $n$ tiles are squares, which can be done $\left(\begin{array}{l}n \\ k\end{array}\right)$ ways. These $n$ tiles have a length of $k+2(n-k)=2 n-k$. We extend this to a $(2 n-1)$-tiling by appending a $(k-1)$-tiling which can be created $f_{k-1}$ ways. Hence, there are $\left(\begin{array}{l}n \\ k\end{array}\right) f_{k-1}$ tilings with $k$ squares among the first $n$ tiles. Altogether, the number of $(2 n-1)$-tilings is $\sum_{k \geq 0}\left(\begin{array}{l}n \\ k\end{array}\right) f_{k-1}$ as desired.

With this logic in mind, we can combinatorially prove similar, more complicated Fibonacci identities. For combinatorial convenience, we state these using $f_{n}$ notation. The next two identities were suggested to us by Benoit Cloitre.

Identity 2. For $n \geq 0$,

$$
\sum_{k \geq 0}\left(\begin{array}{l}
n \\
k
\end{array}\right) f_{3 k-1}=2^{n} f_{2 n-1} .
$$

Proof: Like before, we let $S$ be the set of $(2 n-1)$-tilings, but here we assign a color to each of the first $n$ tiles, white or black. Clearly, $S$ has size $2^{n} f_{2 n-1}$, the right side of Identity 2 .

On the left side of the identity, we generate elements of $S$ by first choosing $n-k$ of the first $n$ tiles to be white dominoes, which can be done $\left(\begin{array}{l}n \\ k\end{array}\right)$ ways, and then somehow using a $(3 k-1)$-tiling to determine the rest of the $(2 n-1)$-tiling.

To do this mapping, we let $X$ be an arbitrary (uncolored) $(3 k-1)$-tiling. Using $X$, we determine the rest of the $(2 n-1)$-tiling (where the first $k$ tiles will be white squares, black squares, or black dominoes) as follows: identify the first $k$ blocks of $X$, where a block has length two if it is ss or $d$ and has length three if it is $s d$. For example, when 
$k=4$, the first four blocks of the 11-tiling ssdsddss are $s s, d, s d, d$. The rest of the tiling is called the tail of $X$ (which would be $s s$ in our example). Notice that a (3k-1)-tiling will always have at least $k$ blocks, and the tail could be empty.

If we label these blocks in order from 1 to $k$, then these $k$ blocks will be mapped to the $k$ colored tiles that are not white dominoes determined by the $\left(\begin{array}{l}n \\ k\end{array}\right)$ term. Specifically, we map each "2-block" to a colored square and each "3-block" to a colored domino as in Figure 2 .

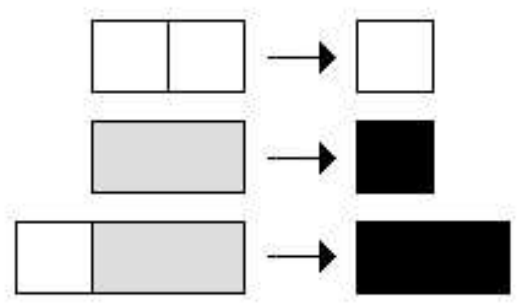

Figure 2: 2,3 block mappings. Blocks of size two and three are mapped to colored squares and black dominoes respectively.

The tail of $X$ is simply mapped "as is," representing the uncolored portion of the $(2 n-1)$-tiling. For example, when $n=6$ and $k=4$, our 11-tiling ssdsddss would first be mapped to the colored tiling $f(X)$, as in Figure 3.

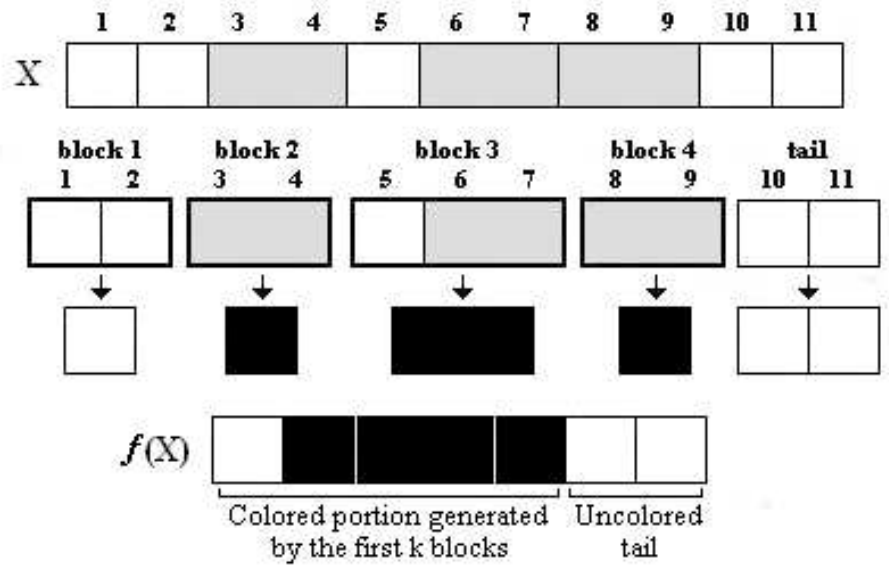

Figure 3: With $k=4$, these $3 k-1$ tiles generate the white squares, black squares and black dominoes, along with the uncolored tail in $S$.

Next, to complete the $(2 n-1)$-tiling, we insert the white dominoes in their prescribed positions. For example, if our white dominoes were chosen to be tiles 1,4 , and 5 , then our $(2 n-1)$-tiling (with $k=4, n-k=3, n=7$ ) would look like the tiling in Figure 4. 


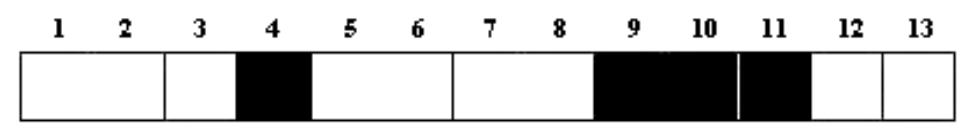

Figure 4: The $(2 n-1)$-tiling is completed by inserting white dominoes among the first $n$ tiles. Here the white dominoes are the first, fourth, and fifth tiles of $S$.

Notice that the length of every block shrinks by one when it is mapped to a colored tile, so if $X$ has length $3 k-1$, then $X$ will be mapped to a tiling $f(X)$ of length $2 k-1$. Combined with the $n-k$ white dominoes, whose locations we know, we achieve a tiling of length $(2 k-1)+2(n-k)=2 n-1$, as desired. This mapping can be easily reversed by selecting an element of $S$, noting the position of the white dominoes and then turning the remaining $k$ colored tiles into blocks to create $X$.

Identity 3. For $n \geq 0$,

$$
\sum_{k \geq 0}\left(\begin{array}{l}
n \\
k
\end{array}\right) f_{4 k-1}=3^{n} f_{2 n-1} .
$$

This identity can be combinatorially proved using the method of Identity 2 . The result will then suggest a generalized identity.

Proof: Like before, we define $S$ to be the set of $(2 n-1)$-tilings, but now each of the first $n$ tiles is colored one of three colors, white, gray, and black. The right side of Identity 2 counts $S$, by definition.

Again, on the left we choose which $n-k$ of the first $n$ tiles will be white dominoes. Then using $X$, an uncolored ( $4 k-1)$-tiling, we generate the rest of the $(2 n-1)$-tiling. Reading $X$ from left to right, we identify the first $k$ blocks of length three or four. A block has length three, unless its of the form $s s d$ or $d d$, in which case it has length four. These $k$ blocks will determine the colored tiles: each 3-block becomes a colored square and each 4-block becomes a colored (non-white) domino. See Figure 5.

The rest of $X$, the tail, is mapped "as is," representing the uncolored portion of the $(2 n-1)$-tiling. For example, suppose $k=5$, and suppose our $(4 k-1)$-tiling is the 19-tiling $d s s d d d s s s s d s d$. Our first five blocks would be $d s, s d, d d$, sss and sd and our tail would be $s d$.

In this case, every block shrinks by two when it is mapped to a colored tile, so if $X$ has length $4 k-1$, then $X$ will be mapped to a tiling of $f(X)$ of length $2 k-1$. Again, combined with the $n-k$ white dominoes, whose locations we know, we achieve a tiling of length $(2 k-1)+2(n-k)=2 n-1$, as desired. This mapping can be easily reversed. Given an element of $S$, note the position of the white dominoes, then turn the $k$ colored tiles into $k$ blocks to create $X$. 


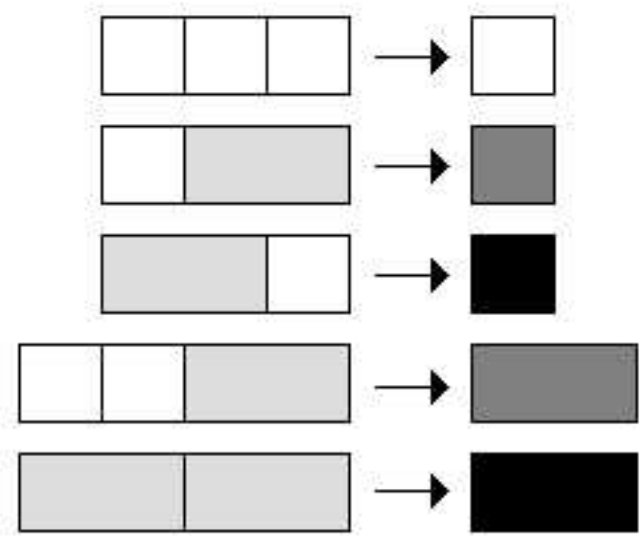

Figure 5: 3,4 block mappings

Upon further investigation of the construction used to prove Identities 1 and 2, we reach the following generalized identity.

Identity 4. For $n \geq 1, q \geq 3$

$$
\sum_{k \geq 0}\left(\begin{array}{l}
n \\
k
\end{array}\right) f_{q k-1}\left(f_{q-3}\right)^{n-k}=\left(f_{q-1}\right)^{n} f_{2 n-1} .
$$

Proof: We still define $S$ like before: $S$ is the set of $(2 n-1)$-tilings where each of the first $n$ tiles is colored one of $f_{q-1}$ colors, which we call colors 1 through $f_{q-1}$. Clearly $S$ is counted by the right side of the identity.

To interpret the left side, imagine that colors 1 through $f_{q-3}$ are light colors, and the remaining $f_{q-1}-f_{q-3}=f_{q-2}$ colors are dark. First, choose which $n-k$ of the first $n$ tiles will be light dominoes, and then assign them a color. This can be done $\left(\begin{array}{l}n \\ k\end{array}\right)\left(f_{q-3}\right)^{n-k}$ ways. Next using an uncolored $(q k-1)$-tiling $X$, we determine the rest of the tiling by identifying the first $k$ blocks of size $q-1$ or $q$, along with its tail.

We create our blocks as follows. Try to form blocks of size $q-1$, but if our string is a block of size $q-2$ followed by a domino, we instead create a block of size $q$ (there are $f_{q-2}$ such blocks). Each block of size $q-1$ (there are $f_{q-1}$ blocks of this size) is mapped in 1-1 fashion to create one of the $f_{q-1}$ colored squares. Each block of size $q$ is mapped in 1-1 fashion to one of the $f_{q-2}$ dark dominoes. Since each of the $k$ blocks is mapped to an object of size $q-2$ smaller, then $X$ is converted (along with its tail) to an object of size $(q k-1)-(q-2) k=2 k-1$. Thus, along with the $n-k$ light dominoes chosen at the beginning, the total length is $2(n-k)+(2 k-1)=2 n-1$, as desired.

Notice that setting $q=3$ and 4 results in Identities 2 and 3, respectively. In fact, 
Identity 3 is true for $q \geq 0$, but when $q=0$ or 2 , the identity is trivial, since $f_{-1}=0$. When $q=1$, since $f_{-2}=1$, Identity 3 and its proof reduces to Identity 0 .

This identity can be easily "extended" to shifted Fibonacci sequences. If we let $S$ count colored tilings of length $2 n-1+p$, where $p \geq 0$, but still only color the first $n$ tiles, then the same argument works exactly as before, but now we simply extend the length of $X$ by $p$ cells, all of which will appear in the tail. Thus, without any extra work, we get

Identity 5. For $n \geq 1, q \geq 0, p \geq 0$

$$
\sum_{k \geq 0}\left(\begin{array}{l}
n \\
k
\end{array}\right) f_{q k-1+p}\left(f_{q-3}\right)^{n-k}=\left(f_{q-1}\right)^{n} f_{2 n-1+p} .
$$

Finally, we can generalize this identity to the so called Gibonacci numbers $G_{n}$, defined by the recurrence

$$
G_{n}=G_{n-1}+G_{n-2}, \text { for } n \geq 2
$$

where $G_{0}$ and $G_{1}$ are arbitrary real numbers. In [1], it is shown that $G_{n}$ counts the total weight of all $n$-tilings, where a tiling that ends in a domino has weight $G_{0}$ and a tiling that ends in a square has weight $G_{1}$. In all of our previous arguments, the last tile always occurs in the tail, and so our bijection is "weight preserving." Therefore, we have, after shifting the index by $p$,

Identity 6. For $n \geq 1, q \geq 0, p \geq 1$,

$$
\sum_{k \geq 0}\left(\begin{array}{l}
n \\
k
\end{array}\right) G_{q k+p}\left(f_{q-3}\right)^{n-k}=\left(f_{q-1}\right)^{n} G_{2 n+p} .
$$

In fact, since a shifted Gibonacci sequence is simply another Gibonacci sequence with different initial conditions, Identity 6 is true even when $p \leq 0$. Hence, setting $p=0$, the following corollary is just as strong as Identity 6 .

Corollary 7. For $n \geq 1, q \geq 0$,

$$
\sum_{k \geq 0}\left(\begin{array}{l}
n \\
k
\end{array}\right) G_{q k}\left(f_{q-3}\right)^{n-k}=\left(f_{q-1}\right)^{n} G_{2 n}
$$

or, equivalently, using the classical Fibonacci numbers $F_{j+1}=f_{j}$,

$$
\sum_{k \geq 0}\left(\begin{array}{l}
n \\
k
\end{array}\right) G_{q k}\left(F_{q-2}\right)^{n-k}=\left(F_{q}\right)^{n} G_{2 n}
$$

With the tools we have used to perform the previous bijections, we can now examine the first eight "unaccounted" Vajda identites, which we label as V69 through V76. (Ac- 
tually, V69 was recently proved by Zeilberger [3] by counting walks on a graph, but we shall take a different approach.) The first identity looks strikingly similar to Identity 1 :

V69 For $n \geq 0$,

$$
\sum_{k \geq 0}\left(\begin{array}{c}
2 n \\
k
\end{array}\right) f_{2 k}=5^{n} f_{2 n} .
$$

Proof: We will approach this problem in a familiar fashion. We define $S$ to be the set of $2 n$-tilings where the first $n$ tiles are each assigned one of five colors. The number of such tilings is clearly $5^{n} f_{2 n}$, which is the right side of our identity.

We now define $T$ to be the set of $4 n$-tilings with the feature that the first $2 n$ tiles have 2 different colors for dominoes, white and black. (We shall denote white dominoes with $w$, black dominoes with $b$, and squares with $s$.) The natural way to construct objects in $T$ is to first choose, for some $0 \leq k \leq 2 n, 2 n-k$ of the first $2 n$ tiles to be black dominoes, which can be done $\left(\begin{array}{c}2 n \\ k\end{array}\right)$ ways, and to fill the remainder with an uncolored (i.e., all white squares and dominoes) $2 k$-tiling. (Note that $2(2 n-k)+2 k=4 n$.) Summing over all possible values of $k$, gives us the size of $T$, as given by the left side of our identity. It remains to find a bijection between $T$ and $S$.

Let $X$ be a $4 n$-tiling from $T$. We denote the first $2 n$ tiles as the colored portion of $X$, and the rest of the tiling is called the tail of $X$. Notice that the length of the tail is equal to the number of squares among the first $2 n$ tiles of $X$. We will use the first $n$ pairs (and some of the first few tiles in the tail) to determine the $n$ colored tiles of the $2 n$-tiling of $S$. The rest of the tail will be mapped "as is." More precisely, pairs of length 3 will map to colored squares and pairs of length 4 will map to colored dominoes. In particular, we generate all colored tiles with colors 1 through 4 as in Figure 6.
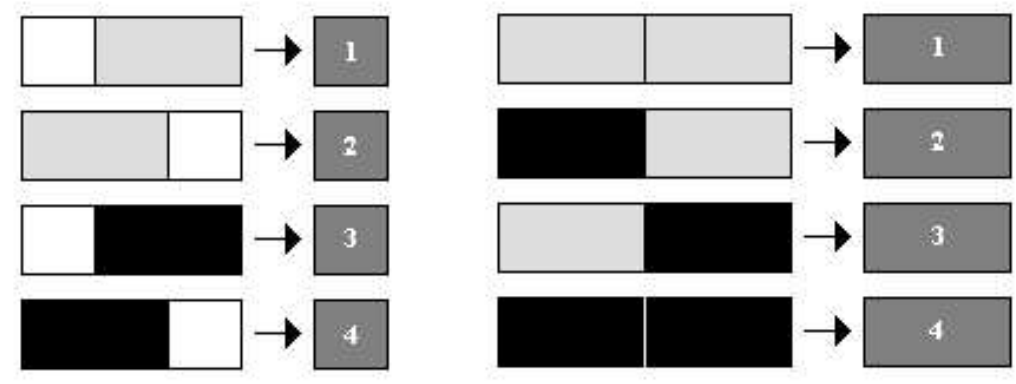

Figure 6: Blocks of length three and four are respectively mapped to squares and dominoes using colors 1 through 4 .

What about the $s^{2}$ pair (consecutive squares)? These will be used to generate a tile of color 5 , but should it be a square or a domino? Before we answer that, let's first illustrate our mapping when we have no $s^{2}$ pairs. For $n=3$, in the 12-tiling in Figure 7 , we have $2 n-k=3$ black dominoes among the first six tiles. 


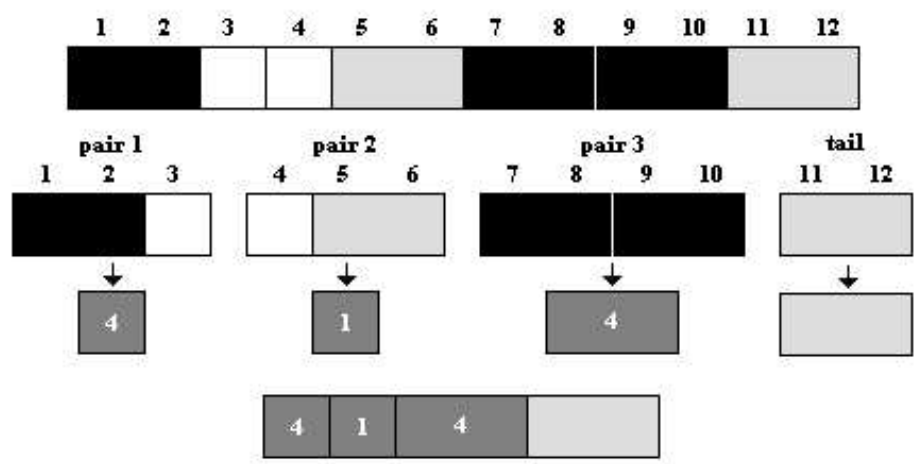

Figure 7: A mapping from $T$ to $S$ with no ss blocks. The output will have no tiles of color 5 .

We deal with the $s^{2}$ pairs as follows. Recall that the length of the tail is equal to the number of squares among the first $2 n$ tiles. Hence if our first $2 n$ tiles contain $m s^{2}$ blocks, then the length of the tail must be at least $2 m$, so the tail has at least $m$ tiles. Hence for the first $s^{2}$ pair we encounter, we look at the first tile of the tail. If that tile is a square, then we extend our $s^{2}$ block to an $s^{3}$ block (of length three) and map it to a square of color 5. If that tile is a domino (necessarily white, since it belongs in the tail), then we extend $s^{2}$ to $s^{2} w$ (which has length 4 ) and map it to a domino of color 5 . This is illustrated in Figure 8.
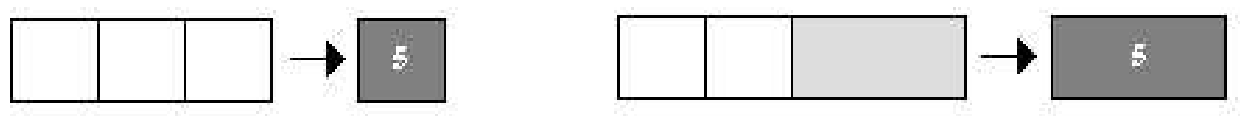

Figure 8: For the $i$-th $s^{2}$ block, we let the $i$-th tile of the tail, tile $2 n+i$, determine which type of color 5 tile to generate.

In general, if our tiling has $j$ blocks of type $s^{2}$, then for each $1 \leq i \leq j$, the $i$-th $s^{2}$ block is extended to $s^{3}$ or $s^{2} w$, depending on the $i$-th tile of the tail (tile $2 n+i$ ). The remainder of our tail, beginning with tile $2 n+j+1$, will be mapped as is. Note that every block has length three or four and is mapped to a colored tile of length one or two, respectively. Hence the colored tiling will have length $4 n-2 n=2 n$.

For example, suppose $n=3$ and we have the 12-tiling ssbwsssws. (See Figure 9.) The 3 tiling pairs would be $s s, b w$, and $s s$. For the first $s s$, we take the first tile in the tail, $s$, and append it. Likewise, for the second $s s$, we take the second tile in the tail, $w$, and append it. As a result, we would have the tiling blocks sss, bw, and ssw with a remainder of $s$. This would map, as in Figure 9, as follows: 

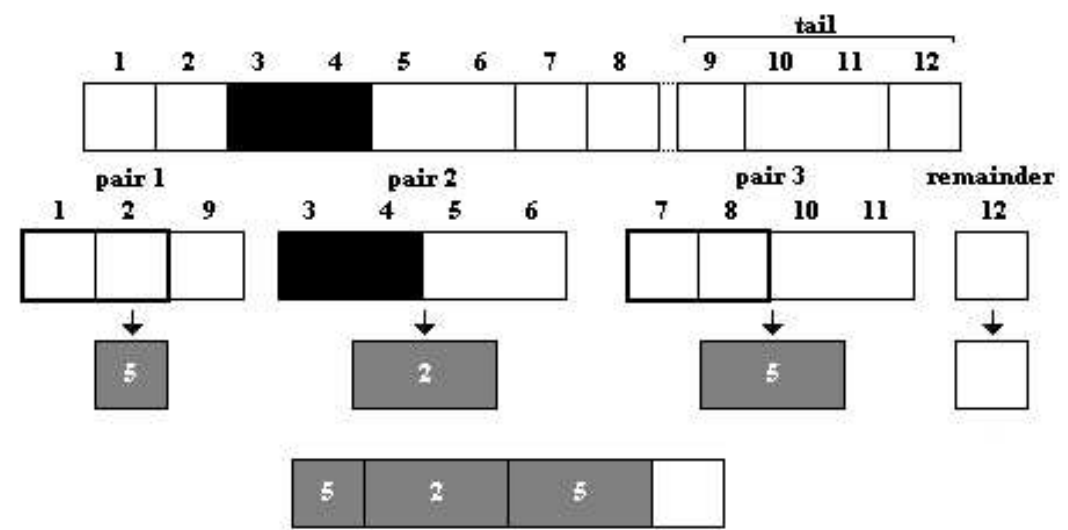

Figure 9: A mapping from $T$ to $S$ that uses ss blocks will generate tiles of color 5 .

This process can be easily reversed. Simply map each colored tile in the $2 n$ board to its corresponding tiling pair, noting that the tiles of color 5 map to sss and $s s w$, where the third tile is part of the tail. Finally, the uncolored portion becomes the remainder of the tail to complete the $4 n$ board.

The mapping used in Identity V69 can also be applied to Fibonacci sequences that have been shifted by $p$. This can easily be incorporated into the above bijection: by adding $p$ we simply have a longer tail, the portion of the tiling which is simply appended during the mapping. That is, we instantly have

Corollary V69a For $n \geq 0$,

$$
\sum_{k \geq 0}\left(\begin{array}{c}
2 n \\
k
\end{array}\right) f_{2 k+p}=5^{n} f_{2 n+p}
$$

Additionally, by giving the last tile of the tail a weight, we can generalize Identity V69 to apply to any Gibonacci sequence since the tail is simply appended during the mapping. The bijection is weight-preserving since the weighted tile will not be involved in any tiling pair or colored tile. This gives us the generalized identity

V69b For $n \geq 0, p \geq 0$

$$
\sum_{k \geq 0}\left(\begin{array}{c}
2 n \\
k
\end{array}\right) G_{2 k+p}=5^{n} G_{2 n+p}
$$

and since shifting a Gibonacci sequence by $p$ simply produces a new Gibonacci sequence, Identity $\mathrm{V} 69 \mathrm{~b}$ is true even when $p<0$. Thus it is more elegant, and just as general, to say 
V69c For $n \geq 0$,

$$
\sum_{k \geq 0}\left(\begin{array}{c}
2 n \\
k
\end{array}\right) G_{2 k}=5^{n} G_{2 n}
$$

Identity V71 comes as an immediate corollary to V69c when $G_{n}=L_{n}$.

V71 For $n \geq 0$

$$
\sum_{k \geq 0}\left(\begin{array}{c}
2 n \\
k
\end{array}\right) L_{2 k}=5^{n} L_{2 n}
$$

The next two Vajda identities

V70 For $n \geq 0$,

$$
\sum_{k \geq 0}\left(\begin{array}{c}
2 n+1 \\
k
\end{array}\right) F_{2 k}=5^{n} L_{2 n+1}
$$

and

V72 For $n \geq 0$

$$
\sum_{k \geq 0}\left(\begin{array}{c}
2 n+1 \\
k
\end{array}\right) L_{2 k}=5^{n+1} F_{2 n+1}
$$

are corollaries of the following Gibonacci identity:

V70a For $n \geq 0$,

$$
\sum_{k \geq 0}\left(\begin{array}{c}
2 n+1 \\
k
\end{array}\right) G_{2 k}=5^{n}\left(G_{2 n}+G_{2 n+2}\right) .
$$

Proof:

$$
\begin{aligned}
\sum_{k \geq 0}\left(\begin{array}{c}
2 n+1 \\
k
\end{array}\right) G_{2 k} & =\sum_{k \geq 0}\left(\begin{array}{c}
2 n \\
k
\end{array}\right) G_{2 k}+\sum_{k \geq 0}\left(\begin{array}{c}
2 n \\
k-1
\end{array}\right) G_{2 k} \\
& =\sum_{k \geq 0}\left(\begin{array}{c}
2 n \\
k
\end{array}\right) G_{2 k}+\sum_{j \geq 0}\left(\begin{array}{c}
2 n \\
j
\end{array}\right) G_{2 j+2} \\
& =5^{n}\left(G_{2 n}+G_{2 n+2}\right),
\end{aligned}
$$

where the last equality follows by applying V69c and V69b (with $p=2$ ).

The algebraic argument above can be "combinatorialized" by mapping a board of length $4 n+2$ (where the first $2 n+1$ tiles may use black or white dominoes) to a colored tiling of length $2 n$ or $2 n+2$, by considering tile $2 n+1$. If that tile is a black domino, we delete it, and create the colored tiling of length $2 n$ as before. If that tile is white, we just make it the first tile of the tail and generate a $(2 n+2)$-tiling with $n$ colored tiles.

Identities V70 and V72 now follow by setting $G=F$ and $G=L$, respectively, along with the identities $F_{2 n}+F_{2 n+2}=L_{2 n+1}$ and $L_{2 n}+L_{2 n+2}=5 F_{2 n+1}$ (which also have 
elementary combinatorial proofs, given in [1]).

The remaining four Vajda identities are given below.

V73 For $n \geq 1$,

$$
\sum_{k \geq 0}\left(\begin{array}{c}
2 n \\
k
\end{array}\right) F_{k}^{2}=5^{n-1} L_{2 n}
$$

V74 For $n \geq 0$,

$$
\sum_{k \geq 0}\left(\begin{array}{c}
2 n+1 \\
k
\end{array}\right) F_{k}^{2}=5^{n} F_{2 n+1}
$$

V75 For $n \geq 0$,

$$
\sum_{k \geq 0}\left(\begin{array}{c}
2 n \\
k
\end{array}\right) L_{k}^{2}=5^{n} L_{2 n}
$$

V76 For $n \geq 0$,

$$
\sum_{k \geq 0}\left(\begin{array}{c}
2 n+1 \\
k
\end{array}\right) L_{k}^{2}=5^{n+1} F_{2 n+1}
$$

Each of these identities can be proved (and generalized) using the mapping developed for V69.

We begin by proving a variation of Identity V74, using the combinatorially convenient quantity $f_{k}=F_{k+1}$.

V74a For $n \geq 0$,

$$
\sum_{k \geq 0}\left(\begin{array}{c}
2 n+1 \\
k
\end{array}\right) f_{k}^{2}=5^{n} f_{2 n+2}
$$

Proof: Our strategy is to combinatorially prove

$$
\sum_{k \geq 0}\left(\begin{array}{c}
2 n+1 \\
k
\end{array}\right) f_{k}^{2}=\sum_{k \geq 0}\left(\begin{array}{c}
2 n \\
k
\end{array}\right) f_{2 k+2}
$$

then from the mapping of Identity V69a, we immediately obtain $5^{n} f_{2 n+2}$.

As in the proof of V69, the set of objects on the right, which we denote by $S$, are $(4 n+2)$-tilings that allow black dominoes among the first $2 n$ tiles. The set of objects on the left, denoted by $T$, count $(4 n+2)$-tilings that allow black dominoes (say $2 n+1-k$ of them) among the first $2 n+1$ tiles, but the remaining white tiles ( $2 k$ of them) must form a tiling that is breakable in the middle (since a $2 k$-tiling that is breakable at cell $k$ can be created $f_{k}^{2}$ ways). Let $A$ be a tiling from $T$. If tile $2 n+1$ is white, then $A$ also belongs to $S$, and we map $A$ to itself. If tile $2 n+1$ is a black domino, then we map it to an element of $S$ by moving the black domino to the middle of the breakable white tiling, then changing its color to white. (If the white tiling had length $2 k$, then it was breakable at $k$. But after the move, it has length $2 k+2$, but is not breakable at $k+1$.) This is a bijection from $T$ to $S$, as desired. 
This argument can be "extended" (by considering tilings of length $4 n+2+2 p$ that end with a weighted tile that allow black dominoes among the first $2 n$ or $2 n+1$ tiles) to show that for $p \geq-1$,

$$
\sum_{k \geq 0}\left(\begin{array}{c}
2 n+1 \\
k
\end{array}\right) f_{k+p} G_{k+p}=\sum_{k \geq 0}\left(\begin{array}{c}
2 n \\
k
\end{array}\right) G_{2 k+2 p+2},
$$

and therefore by Identity V69b,

$$
\sum_{k \geq 0}\left(\begin{array}{c}
2 n+1 \\
k
\end{array}\right) f_{k+p} G_{k+p}=5^{n} G_{2 n+2 p+2} .
$$

Identity V74 now follows immediately by letting $p=-1$ and $G=f$.

Another way to write this identity, after replacing $n$ with $n-1$ is

$$
\sum_{k \geq 0}\left(\begin{array}{c}
2 n-1 \\
k
\end{array}\right) f_{k+p} G_{k+p}=5^{n-1} G_{2 n+2 p}
$$

or more elegantly as

V74b For $n \geq 0$,

$$
\sum_{k \geq 0}\left(\begin{array}{c}
2 n-1 \\
k
\end{array}\right) f_{k+p} G_{k}=5^{n-1} G_{2 n+p}
$$

We may now derive V73 by first showing the more general identity V73a For $n \geq 0$,

$$
\sum_{k \geq 0}\left(\begin{array}{c}
2 n \\
k
\end{array}\right) f_{k+p} G_{k}=5^{n-1}\left(G_{2 n+p}+G_{2 n+p+2}\right) .
$$

Proof: Our proof is a simple algebraic manipulation of Identity V74b, but the argument could easily be made combinatorial by considering tile $2 n$.

$$
\begin{aligned}
\sum_{k \geq 0}\left(\begin{array}{c}
2 n \\
k
\end{array}\right) f_{k+p} G_{k} & =\sum_{k \geq 0}\left(\begin{array}{c}
2 n-1 \\
k
\end{array}\right) f_{k+p} G_{k}+\sum_{k \geq 0}\left(\begin{array}{c}
2 n-1 \\
k-1
\end{array}\right) f_{k+p} G_{k} \\
& =\sum_{k \geq 0}\left(\begin{array}{c}
2 n-1 \\
k
\end{array}\right) f_{k+p} G_{k}+\sum_{j \geq 0}\left(\begin{array}{c}
2 n-1 \\
j
\end{array}\right) f_{j+(p+1)} H_{j}, \text { where } H_{j}=G_{j+1} \\
& =5^{n-1} G_{2 n+p}+5^{n-1} H_{2 n+p+1} \\
& =5^{n-1}\left(G_{2 n+p}+G_{2 n+p+2}\right) .
\end{aligned}
$$

Identity V73 now follows by setting $p=-1, G=F$, and using $F_{2 n-1}+F_{2 n+1}=L_{2 n}$. Also, by setting $p=-1, G=L$ and using $L_{2 n-1}+L_{2 n+1}=5 F_{2 n}$, we get the identity 
V73b For $n \geq 0$,

$$
\sum_{k \geq 0}\left(\begin{array}{c}
2 n \\
k
\end{array}\right) F_{k} L_{k}=5^{n} F_{2 n}
$$

We now generalize Identities V75 and V76 using the identity

$$
L_{k} G_{k}=G_{2 k}+(-1)^{k} G_{0},
$$

which has a simple combinatorial proof (given as Identity 45 in [1], page 28). Combining this with Identity V69c immediately produces

V75a

$$
\sum_{k \geq 0}\left(\begin{array}{c}
2 n \\
k
\end{array}\right) L_{k} G_{k}=5^{n} G_{2 n}
$$

Setting $G=L$ gives us Identity V75, and setting $G=F$ gives us Identity V73b again. Likewise, by applying Identity V70a, we have

V76a

$$
\sum_{k \geq 0}\left(\begin{array}{c}
2 n+1 \\
k
\end{array}\right) L_{k} G_{k}=5^{n}\left(G_{2 n}+G_{2 n+2}\right),
$$

which reduces to Identity V76 when $G=L$.

As this paper was being prepared, Alex Eustis combinatorially proved and generalized the 100th uncounted Vajda Identity (V106), the continued fraction identity:

$$
\frac{F_{(t+1) m}}{F_{t m}}=L_{m}-\frac{(-1)^{m}}{L_{m}-\frac{(-1)^{m}}{L_{m}-\frac{(-1)^{m}}{\ddots-\frac{(-1)^{m}}{L_{m}}}}},
$$

where the number $L_{m}$ appears $t$ times. But the details of that will have to be continued at another time.

\section{References}

[1] A. T. Benjamin and J. J. Quinn, Proofs That Really Count: The Art of Combinatorial Proof, The Dolciani Mathematical Expositions, 27, Mathematical Association of America, Washington, DC, 2003

[2] S. Vajda, Fibonacci \& Lucas Numbers, and the Golden Section: Theory and Applications, Wiley \& Sons, Inc., New York, 1989.

[3] D. Zeilberger, A Fibonacci-Counting Proof Begged by Benjamin and Quinn, to appear in Applications of Fibonacci Numbers, Volume 10, (William Webb, ed.), Kluwer Academic Publishers, 2006. 